\title{
Pratiques alimentaires et suivi des enfants malnutris aigus sévères à Koutiala en 2015 au Mali
}

\section{Eating practices and monitoring of severe acute malnourished children in Koutiala in 2015 in Mali}

\author{
Oumar SANGHO ${ }^{1,2,3^{*}}$, Borodjan DIARRA ${ }^{4}$, Ndeye Lallah Nina KOITE ${ }^{1,2}$, Dassouni COULIBALY ${ }^{2}$, Fanta SANGHO ${ }^{1,2,5}$, Aboubacar \\ SANGHO $^{6}$, Cheick Abou COULIBALY ${ }^{2}$, Haoua DEMBELE ${ }^{7}$, Mariam TRAORE ${ }^{7}$, Assa SIDIBE ${ }^{7}$, Djénéba COULIBALY2,7, Baba
} DIALLO ${ }^{8}$, Samba DIOP², Akory AG IKNANE ${ }^{1,2,7}$

1. Département d'Enseignement et de Recherche des Sciences Biologiques et Médicales, Faculté de Pharmacie (FAPH), Université des Sciences, des Techniques et des Technologies de Bamako (USTTB), Mali.

2. Département d'Enseignement et de Recherche en Santé Publique et Spécialités, FMOS, USTTB, Mali.

3. Agence Nationale de Télésanté et d'Informatique Médicale, Bamako, Mali.

4. Direction Générale de la Santé et de l'Hygiène Publique (DGSHP), Sous-Direction des Etablissements Sanitaires et Réglementation (SDESR) Bamako Mali.

5. Direction de la Pharmacie et du Médicament (DPM), Bamako, Mali. 6. Département d'Enseignement et de Recherche des Sciences Pharmaceutiques, FAPH/USTTB, Mali

7. Institut National de la Santé Publique (INSP), Bamako, Mali. 8. Centre Hospitalier Universitaire, Centre National d'OdontoStomatologie (CNOS), Bamako, Mali.

*Auteur correspondant : Dr Oumar SANGHO, MD, DIU EPIVAC MPH, PhD - Epidemiology, Maître-Assistant en Epidémiologie au DER des Sciences Biologiques et Médicales, FAPH/USTTB/Mali, Chef Section Planifications et Etudes à l'Agence Nationale de Télésanté et d'IIformatique Médicale (ANTIM), Tél Mobile : (00223) 76225877 / 66 7667 82, oumarsangho2005@yahoo.fr

\section{Résumé}

Introduction: Plus de 20 millions d'enfants souffrent de malnutrition aigüe dans les pays en développement. Elle constitue un problème de santé publique. L'objectif visait à étudier les pratiques alimentaires, le suivi nutritionnel et les facteurs associés chez les enfants malnutris aigus sévères à Koutiala. Matériel et méthodes : De Juillet à Aout 2015, une enquête transversale exhaustive a été menée auprès d'un échantillon de 238 enfants de 06 à 59 mois au centre de santé de référence de Koutiala. Les mesures anthropométriques et le test diagnostic rapide du paludisme ont été effectués. EPI DATA 3.5.1 et SPSS 23.0 ont été utilisés pour la saisie et l'analyse des données. Résultats : Les enfants de 06 à 24 mois représentaient $42,8 \%$ dont $51,3 \%$ étaient des filles. $55 \%$ des enfants étaient marastiques. Une récupération de moins de 4 jours est observée chez $48,3 \%$ des malnutris et $39,9 \%$ des enfants étaient mal sevrés. Conclusion : Cette étude a révélé chez les enfants un sevrage précoce (arrêt trop tôt du lait maternel au profit d'autres aliments de complément), des pratiques alimentaires inadéquates. On notait un meilleur suivi avec un taux de récupération élevé.

Mots clés : Pratique alimentaire, enfants malnutris, Koutiala, Sikasso, Mali.

\section{Abstract}

Introduction: More than 20 million children suffer from acute malnutrition in developing countries, it is a public health problem. The objective was to study the feeding practices and nutritional monitoring of severely acute malnourished children in Koutiala in order to identify the factors linked to it. Materials and Material and methods: this was a descriptive cross-sectional study in malnourished children from 06 to 59 months seen at the CSRéf in Koutiala from July 1 to August 31,2015 . The data were collected, entered in EPI Data 3.5.1, transferred to ENA for SMART and analyzed on SPSS 23.0. Results: The most represented age group was 06 to 24 months $(42.8 \%)$. Girls were more represented $(51.3 \%)$. The most represented form was the $55 \%$ slump. It was found that $48.3 \%$ of the malnourished were recovered within 4 days, $(39.9 \%)$ of the children were weaned abruptly. Conclusion: the nutritional status of children aged 6 to 59 months was worrying, a high rate of early weaning. The quality of follow-up was satisfactory with a high rate of treatment success. Food practices were inadequate.

Key words: Food practice, malnourished children, Koutiala, Sikasso, Mali.

\section{INTRODUCTION}

La santé de l'homme dépend en grande partie de la manière dont il se nourrit, car un régime alimentaire déséquilibré peut être aussi néfaste qu'un manque de nourriture (1). La malnutrition est un état pathologique résultant de la carence ou de l'excès, relatif ou absolu d'un ou de plusieurs nutriments essentiels, que cet état se manifeste cliniquement ou ne soit décelable que par des analyses biochimique, anthropométrique ou physiologique (1). Sans une nutrition adéquate, les enfants ne pourront développer tout leur potentiel et les adultes rencontreront des difficultés à conserver ou élargir le leur (1).

Le retard de croissance, l'émaciation et les carences en micronutriments sont estimés être à l'origine de près de 3,1 millions de décès d'enfants chaque année (2).

La malnutrition est la maladie carencielle la plus répandue dans le monde en développement. Elle résulte aussi des pratiques alimentaires inadéquates concernent non seulement la qualité des aliments donnés aux enfants, mais aussi aux étapes de leurs introductions dans son alimentation notamment le sevrage (3). Le terme " sevrage " vient du latin SEPARARE, qui signifie " séparer ». Le sevrage de l'allaitement est une phase naturelle et inévitable du développement de l'enfant. C'est un processus complexe qui exige des rajustements nutritionnels, immunologiques, biochimiques et psychologiques (4). Le sevrage peut signifier l'arrêt complet de l'allaitement (le sevrage « abrupt » ou définitiff ou, pour les besoins du 
présent énoncé, le début du processus graduel d'introduction d'aliments complémentaires dans le régime du nourrisson. L'introduction du tout premier aliment autre que du lait maternel constitue, par définition, le véritable commencement du sevrage. (4). Le sevrage brutal est l'arrêt total et définitif de l'allaitement au profit de l'alimentation de complément en dehors de tout processus ou préparation.

La région de Sikasso, dans laquelle fait partie le cercle de Koutiala qui est de plus en plus pauvre en denrées de première nécessité au profit de la culture massive du coton) (5), a connu, en 2010 , un niveau de prévalence de malnutrition élevée, d'émaciation $(11,1 \%)$ supérieur au seuil d'alerte de l'OMS (10\%), de retard de croissance $(51,5 \%)$ supérieur au seuil d'alerte de l'OMS (35\%), d'insuffisance pondérale (34,6\%) (3).

Plus de 20 millions d'enfants souffrent de malnutrition aigüe dans les pays en développement, La malnutrition constitue une priorité du fait de l'association concomitante d'infections répétées et prolongées chez les enfants de moins cinq ans. La malnutrition constitue l'un des principaux problèmes de santé et de bien-être qui affectent les enfants dans les pays en développement en général et au Mali en particulier (3). L'alimentation des mères et celle des enfants sont étroitement liées, la malnutrition commence souvent dès la conception et les troubles qui en résultent apparaissent déjà dès la 2ème année de vie de l'enfant (6).

La malnutrition affaiblit le système immunitaire et aggrave les maladies (6).

Les enfants ayant un faible poids de naissance sont susceptibles de développer un retard de croissance et à l'âge adulte, ces individus sont exposés à un risque élève de maladies coronariennes, de diabète et de l'hypertension (1).

De plus, les femmes ayant subi un retard de croissance seront prédisposées à perpétuer le cercle vicieux de la malnutrition (1).

Des carences en micronutriments spécifiques comme la vitamine $A$, le fer et l'iode sont répandues et ont des effets néfastes sur la santé (7). Au Mali, $(38 \%$ des enfants) accusent un retard de croissance dont $19 \%$ de forme sévère, $26 \%$ souffrent d'une insuffisance pondérale dont $9 \%$ de forme sévère et $13 \%$ accusent une émaciation dont $5 \%$ de forme sévère (9). En 2014 au moins 995.000 enfants de moins de 5 ans souffraient de malnutrition chronique au Mali (8).

Seulement un tiers des enfants de moins de 06 mois (33\%) sont exclusivement allaités. Les aliments de complément ne sont pas introduits de façon appropriée : seulement moins de la moitié des enfants de 06-09 mois sont toujours allaités et reçoivent des aliments solides ou semi solides (49\%). Seulement, $(8 \%)$ des enfants de 0623 mois sont nourris de façon appropriée selon les trois pratiques optimales d'alimentation du nourrisson et du jeune enfant (9).
Environ 200 millions d'enfants de moins de cinq ans présentent des symptômes aigus ou chroniques de malnutrition et ce nombre s'accroît au cours des pénuries alimentaires saisonnières ainsi que pendant les périodes de famine et de conflits sociaux (10). Selon certaines estimations, la malnutrition est un facteur déterminant pour les quelques treize millions d'enfants de moins de cinq ans qui meurent chaque année de maladies et d'infections que l'on pourrait éviter comme la diarrhée, le paludisme et la pneumonie, ou de plusieurs de ces maladies combinées. Les enfants qui survivent de la malnutrition ont une capacité d'apprentissage réduite et une faible productibilité lorsqu'ils atteignent l'âge adulte, d'où la malnutrition diminue la qualité de vie et entraine des pertes financières importantes dans les familles, les communautés et les pays (10).

Pour mettre un terme à la faim, il faut nécessairement commencer par assurer une production et une distribution de nourritures suffisantes pour tous. Cependant, le seul fait de produire suffisamment ne garantit pas l'éradication de la faim. II faut garantir à tous et à tout moment l'accès alimentaire, c'est à dire l'accès à une nourriture suffisante, saine et adéquate sur le plan nutritionnel, pour une vie saine et active. Malgré les multiples efforts consentis par l'état et ses partenaires dans la lutte contre la malnutrition, le niveau de la malnutrition reste de nos jours très élevé dans notre pays et particulièrement au sein de la population rurale. D'où cette présente étude dont l'objectif visait à étudier les pratiques alimentaires, le suivi nutritionnel et les facteurs associés chez les enfants malnutris aigus sévères à Koutiala.

\section{Matériel et méthodes}

Une enquête transversale descriptive a été menée auprès d'enfants âgés de 06 à 59 ans mois dans le centre de santé de référence (CSRéf) de Koutiala, région de Sikasso au Mali. Tous les enfants de 06 à 59 mois malnutris ayant bénéficié d'une prise en charge médicale au CSRéf et dont les mères ou tuteurs ont accepté de participer à l'enquête, allant du 1er Juillet au 31 Août 2015, ont été inclus dans l'étude. Les données ont été collectées sur un questionnaire pré-testé avant le début de l'enquête.

L'état nutritionnel a été évalué en prenant des mesures anthropométriques, selon les normes de l'OMS. Le test du paludisme a été faite à l'aide du Test Diagnostic Rapide de Paludisme (TDR).

Les données ont été saisies sur EPI Data 3.5.1 et transférées sur ENA for SMART pour la normalisation anthropométrique. Elles ont été ensuite analysées sur SPSS 23.0. Les résultats sont présentés sous forme de tableaux et de graphiques à travers les logiciels Excel 2007 et SPSS 23.0. Le document a été rédigé à l'aide du logiciel Microsoft Word 2007. Une analyse descriptive usuelle a été faite pour décrire la distribution de fréquences, moyennes avec écarts type. 
La proportion de la malnutrition a été estimée chez tous les enfants de 06 à 59 ans en fonction des catégories sociodémographiques, des habitudes alimentaires à savoir l'âge d'introduction, des antécédents familiaux et personnels. Nous avons effectué des croisements pour trouver des associations statistiquement significatives $(p<0,05)$ à l'aide du test de Khi2.

Les considérations éthiques et déontologiques ont été respectées. Elles ont porté entre autres sur les demandes d'autorisations auprès des autorités administratives sanitaires de Koutiala, au respect strict de la confidentialité et l'obtention du consentement volontaire libre et éclairé des personnes enquêtées. II n'y avait aucun risque pour les enquêtées. Aucune information collectée ne permettait de faire le lien entre l'étude et les patients.

\section{RESULTATS}

Les résultats de la présente étude ont porté sur 238 enfants malnutris répondant à nos critères d'inclusion sur 400 enfants reçus en consultation curative au CSREF de Koutiala pendant la période de l'étude.

\subsection{Les caractéristiques sociodémographiques des} enfants et des mères

Dans notre étude, nous avons trouvé une légère prédominance féminine $51,3 \%$ par rapport aux garçons $47,8 \%$ avec un ratio à 1,05 . Plus de la moitié des enfants malnutris $81,1 \%$ avaient des mères non instruites. La tranche d'âge la plus représentée était celle de 6 à 24 mois avec $42,8 \%$ de l'échantillon.

Sur les 238 enfants enquêtés $(38,7 \%)$ provenaient des 5 CSCOM couverts par l'ONG MSFF et $(61,3 \%)$ des autres CSCOM non couverts par le MSFF.

3.2. Caractéristiques nutritionnelles des 238 enfants Le marasme était la forme de la malnutrition la plus fréquente avec $(55 \%)$ suivi de Kwashiorkor $(34 \%)$ et la forme mixte était seulement $11 \%$.

\subsection{Caractéristiques alimentaires des 238 enfants}

Dans notre étude l'introduction du 1er aliment est observée chez plus de $(85,7 \%)$ des enfants dans la tranche d'âge 6 à 12 mois contre $(13,4 \%)$ chez les moins de 6 mois.

Plus de la moitié $(62,2 \%)$ étaient soumis au régime d'aliments de complément et $(37,8 \%)$ étaient toujours allaités avec une diversification alimentaire. Ces enfants consommaient moins de crudités. Après admission à I'URENI, tous les malnutris étaient alimentés à base d'Aliment Thérapeutique Prêt à l'Emploi (ATPE) uniquement et le lait maternel pour ceux qui l'étaient encore. Au total $(60,1 \%)$ des enfants ont été sevrés spontanément contre $(39,9 \%)$ de façon brutale. La grossesse précoce des mères était la cause de $(24,8 \%)$ de sevrage et $(33,6 \%)$ étaient dus aux maladies. Nous avons constaté que la pratique de l'allaitement exclusif était très faible, soit (15\%). Dans notre étude, nous avons trouvé (4\%) d'interdits alimentaires chez les enfants de 06 à 59 mois.

\subsection{Prévalences des maladies associées à la malnutrition}

Parmi les motifs de consultation chez les enfants de 06 à 59 mois, la fièvre représentait $51,7 \%$ et $27,3 \%$ pour les autres motifs. Par ailleurs, on notait $25 \%$ de diarrhée au moment de leur admission à l'URENI. Cette étude révèle également un faible taux d'enfants vaccinés, soit une proportion de $10,5 \%$

Sur les 84 cas de paludisme présumé, nous avons notifié (44\%) de cas de TDR positifs chez les enfants de 06 à 59 mois. Par rapport aux pathologies rencontrées, le paludisme était retrouvé chez $(35,3 \%)$ des enfants dont (50 cas d'accès simple et 34 cas de forme grave) suivie de la gastro-entérite $(26,5 \%)$ et de la Pneumopathie $(10,1 \%)$. Les autres pathologies rencontrées $(28,15 \%)$ étaient surtout les infections urinaires, les infections $\mathrm{ORL}$, les méningites.

\subsection{Paramètres de Suivi des enfants malnutris}

Par rapport à l'issue de la prise en charge, $48,3 \%$ des enfants malnutris avaient récupéré dans un délai inférieur à 4 jours. Nous n'avons enregistré aucun enfant perdu, au cours du suivi. $41,6 \%$ des malnutris étaient récupérés au-delà de 4 jours.

\subsection{Analyse des facteurs liés à la malnutrition (marasme et palu, marasme et $\mathrm{VIH}$, kwashiorkor et la gastroentérite)}

Nous avons trouvé une différence statistiquement significative observée entre la fréquence du marasme et la tranche d'âge des enfants avec $(p=0,001)$.

Environ $73 \%$ des enfants marasmiques présentaient des vomissements et diarrhées avec une différence statistiquement significative observée entre marasme et la gastroentérite $(p=0,0009)$. Pour les autres variables, nous n'avons pas trouvé de relation statistiquement significative avec le marasme (paludisme, l'infection VIH et le sexe).

Plus de $55 \%$ des enfants œdémateux figuraient dans la tranche d'âge 25 mois et plus avec une différence statistiquement significative $(p=0,001)$. Nous avons trouvé que $23,9 \%$ d'enfants kwashiorkor ont présentés des diarrhées et vomissements avec une différence statistiquement significative ( $p=0,0073$ ). Pour les autres variables, nous n'avons pas trouvé de relation statistiquement significative avec le Kwashiorkor.

\section{DISCUSSION}

4.1. Les caractéristiques sociodémographiques de l'échantillon

Notre étude a révélé une légère prédominance féminine $(51,3 \%)$ par rapport aux garçons $(48,7 \%)$ avec un ratio de 1,05. Ce résultat est comparable à celui de (11) qui avait trouvé à Sikasso un ratio de 0,9. Par contre, l'étude menée par (10) avait trouvé le ratio supérieur à 1 en faveur des garçons. Notre ratio était supérieur à celui de (12) qui est de 0,79 en faveur 
des filles. Cette prédominance serait due à la fragilité et à l'exposition des petites filles dans les foyers.

La tranche d'âge la plus représentée était celle de 06 à 24 mois $(42,8 \%)$ suivie de celle 24 à 34 mois $(32,8 \%)$ des enfants. Cette fréquence est comparable à celle trouvée par (10) dans la région de Sikasso (26,3\%). Cela s'expliquerait par le sevrage qui est fréquent à cette tranche, surtout si c'est mal fait.

\subsection{Par rapport aux différentes maladies rencontrées pouvant influencées l'état de santé des malnutris :}

Sur les 238 enfants de notre étude, (25\%) avaient de la diarrhée à leur admission. Cette prévalence était nettement supérieure à celle de (11) qui est de $(3,7 \%)$ et aussi supérieure à la prévalence de diarrhée $(9 \%)$ retrouvée par (9). Cela pourrait s'expliquer par le fait que notre étude a été réalisée entre juillet et aout correspondant à la saison des pluies pendant laquelle l'insalubrité est grandissante avec les eaux stagnantes. Environ (4\%) des enfants avaient des interdits alimentaires. Nos résultats étaient supérieurs à ceux de (11) qui trouve $(0,7 \%)$ d'interdits. Cette prévalence s'expliquerait par l'attachement de nos populations rurales aux pratiques socioculturelles car la quasi-totalité de ces interdits était des animaux sauvages avec comme raison principale évoquée, le totem.

Notre étude a conclu à 84 cas de paludisme avec anémie soit $(35,3 \%)$ avec une positivité du TDR dans (40\%) des cas. Ce taux est proche de celui de (12) qui obtient $(24,4 \%)$ avec le TDR positif dans $(38,8 \%)$ des cas. Notre résultat était inférieur à celui de (9) qui est de $(52 \%)$ pour les enfants de 6 à 59 mois.

Cette prévalence du paludisme pourrait s'expliquer par la saison des pluies pendant laquelle les mères, occupées par les travaux champêtres, n'arrivent pas à prendre soin correctement des enfants et aussi l'envahissement de l'entourage des concessions par les cultures de maïs, mil, etc....

Les enfants issus de parents non instruis occupaient $(81,1 \%)$ de la population générale.

\subsection{Par rapport au statut vaccinal}

II ressort de l'étude une faible couverture vaccinale avec $(10 \%)$ d'enfants vaccinés contre $(51,3 \%)$ de non vaccinés. Notre résultat était nettement inférieur au taux de vaccination de (13) au plan national avec un taux de (50,6\%) d'enfants complètement vaccinés et ceux de (9) avec la proportion d'enfants complètement vaccinés (42\%) dans la région de Sikasso (14). Cela s'expliquerait par les contraintes des mères et des refus de certains chefs de familles.

\subsection{Par rapport aux pratiques alimentaires}

Sur les 238 enfants enquêtés, l'alimentation de complément était pratiquée chez $(62,2 \%)$ avec comme aliments de base (bouillie, plat familial); donc sont plus exposés aux maladies capables de créer un déséquilibre nutritionnel. Les (37,8\%) étaient encore allaités avec une faible diversification alimentaire.
Cela pourrait s'expliquer, entre autres, par une réduction considérable des denrées à cette période de soudure ou leur cherté.

Par rapport aux pratiques alimentaires sur l'alimentation et l'état nutritionnel des enfants, nous avons constaté que la pratique de l'allaitement exclusif était très faible, soit $(15 \%)$ des cas. Ce résultat est supérieur à celui retrouvé par (11).

De nombreuses mères cessent d'allaiter précocement et sont souvent obligées de passer à d'autres formes de préparations alimentaires, qui peuvent ralentir la croissance si les conditions d'hygiène ne sont pas respectées (15).

Les jeunes enfants (entre 6 mois et 5 ans) sont particulièrement sujets aux infections (paludisme, maladies diarrhéiques, etc....) qui, en réduisant leur appétit et en augmentant leur dépense énergétique, peuvent précipiter ou aggraver la malnutrition.

Dans certaines sociétés humaines, les adultes sont servis les premiers et les jeunes enfants les derniers (16).

La tranche d'âge de 13 à 23 mois constituait la majorité des enfants sevrés soit $(52,3 \%)$. Notre résultat est comparable à celui de (17) qui trouve $(79,5 \%)$ des enfants avec la même tranche d'âge de 24 à 35 mois. Le sevrage précoce était l'un des facteurs déterminants de la malnutrition et certaines maladies des enfants, qui pouvaient être liées à des motifs comme : grossesses multiples et rapprochées de la femme, maladies transmissibles par le lait maternel, alimentation inadéquate de la femme allaitante et souvent la charge et l'occupation de la femme. Les céréales constituaient les aliments les plus consommés aux heures habituelles de repas (matin, midi et soir).

L'étude nationale réalisée (18) montre que les céréales de base sont quotidiennement consommées, tandis que les autres aliments (fruits, légumes, lait, œufs et tubercules) le sont faiblement du fait que : - Ces aliments ne sont pas pris en compte dans les habitudes alimentaires et les modes de consommation, - Le niveau de connaissance sur les apports nutritifs de ces aliments est faible, - Les besoins nutritionnels de chaque catégorie de personnes sont méconnus : par exemple, les besoins en aliments d'une femme enceinte, d'un enfant ou d'une personne âgée. La diversification alimentaire est retardée entre 5 et 6 mois (19).

Nous avons constaté que ces enfants malnutris consommaient moins de crudités qui contiennent sans doute des éléments nutritifs essentiels, indispensables au bon fonctionnent de l'organisme. La consommation de certains aliments essentiels pour la croissance de l'enfant varierait en fonction des familles, ethnies et revenus économiques. L'alimentation de complément des enfants nutritionnellement adéquat est particulièrement importante pour la croissance, le développement, et la prévention de la malnutrition. 
4.5. Par rapport aux formes cliniques de la malnutrition aigüe sévère

Dans notre étude, le marasme a représenté $(55 \%)$, contre $(34,5 \%)$ pour kwashiorkor et $(10,5 \%)$ de forme mixte Kwashiorkor et marasme. En effet, le marasme serait la forme de malnutrition aigüe sévère la plus répandue dans le district sanitaire de Koutiala. Cette prédominance a été également notée à l'Unité de Récupération et d'Education Nutritionnelle (URENI) du centre de santé de Koutiala par (12) qui avait trouvé $(50,5 \%)$ de marasme contre $(29,3 \%)$ de Kwashiorkor. 4.6. Sur le suivi des enfants de 06 à 59 mois.

Sur les 238 enfants, le suivi nutritionnel a concerné l'ensemble des enfants malnutris durant l'étude pendant les deux mois. On a retrouvé $(48,3 \%)$ des enfants récupérés dans un délai inférieur à 4 jours $(2,1 \%)$ de décès, $(8 \%)$ de cas de complications et (6\%) d'enfants récupérés au-délà de 4 jours et 1 cas de non répondant.

\section{Conclusion}

II s'agissait dans cette étude de décrire les pratiques alimentaires et le suivi nutritionnel des enfants malnutris. Les résultats montrent que l'état nutritionnel des enfants de 6 à 59 mois était inquiétant, le taux de marasme à $55 \%$, celui de kwashiorkor à $34,5 \%$, le faible taux de vaccination, le taux élevé de sevrage précoce d'où la nécessité d'entreprendre des stratégies et actions pour améliorer la situation nutritionnelle des enfants et la qualité de vie des populations rurales. La prise en charge nutritionnelle est un problème multisectoriel qui devrait inclure les contributions de l'ensemble des disciplines ou secteurs associés (sécurité nutritionnelle, agriculture, santé pour la nutrition, économie, culture...).

Les principales maladies rencontrées associées à la malnutrition aigüe sévère ont été le paludisme la gastroentérite et la pneumopathie. La qualité du suivi était satisfaisante avec un taux élevé de succès aux traitements. Ces carences touchaient en général les enfants de moins de cinq ans et plus particulièrement l'enfant en âge (06 mois) d'introduction d'alimentation complémentaire.

L'allaitement exclusif chez les moins de 6 mois était faible. Les pratiques alimentaires inadéquates font référence, non seulement à la qualité et à la quantité des aliments donnés aux enfants, mais aussi aux étapes de leur introduction. Les céréales étaient les aliments les plus consommés.Par contre, certains produits locaux (tubercules, fruits, légumes...) étaient peu utilisés et valorisés.

VI. Déclaration de conflit d'intérêt

Les auteurs ne déclarent aucun conflit d'intérêt au cours de cette étude.

VII. Références
1. OULD Ne M.H. Pratiques alimentaires et suivi nutritionnel des enfants malnutris de la commune de Sikasso. Thèse de Médecine FMOS : Bamako : 2012 ; Pages 1 et 2, 82p.

2. ZULFIGAR A.B et al. 2013: Lancet nutrition intervention review group and the maternal and child nutrition study group : 2013 ; page 2.

3. AG IKNANE $A$ et al. Pratiques des mères en matière de santé nutrition des enfants de moins de 5 ans à Sikasso au Mali. MSP tome $3 ; 2013 ; 196 p$.

4. Lawrence R. Breastfeeding: A Guide for the Medical Profession. 5e édition. St Louis: Mosby; 1999. pp. 335pp. 338pp. 343-344. [Google Scholar]

5. Dury S, Bocoum I. Le « paradoxe » de Sikasso (Mali) : pourquoi « produire plus » ne suffit-il pas pour bien nourrir les enfants des familles d'agriculteurs ? 2012. Cah Tirés à part : S. Dury Agric 21 : 324-36. doi :

10.1684/agr.2012.0584

6. NUTRITION ESSENTIELLES, FRENCH. 1998 : Actions essentielles en nutrition (guide pour les responsables des activités de santé) : Paris : Flammarion Médecine-sciences ; 1998.

7. MS. CPS. 2012 : Carte sanitaire du Mali version I tome 2 équipements sanitaires personnels et établissements privés 2012.page 58,258 pages.

8. UNICEF. Bulletin humanitaire OCHA du Mali novembre : 2013 : Page 1, 7 pages. WWW.mocha.org/mali

9. INFO-STAT, CPS/MS, INSTAT, ICF International. 2013 : Enquête Démographique et de Santé du MALI (EDSM V) 2012-2013 : Mai 2013 ; 523p.

10. GUEYE B.Y. Suivi nutritionnel des enfants de 06 à 59 mois et pratiques alimentaires des malnutris des communes de Zangaradougou et Dandresso cercle de Sikasso. Thèse de Médecine : FMOS : Bamako ; 2011 ; $71 \mathrm{p}$.

11. HIMAHOU B .0. Suivi nutritionnel des enfants de 06 à 59 mois et pratiques alimentaires des malnutris de la commune rurale de Primpéran dans le cercle de Sikasso. Thèse de Médecine: Bamako ; 2014 ; 100p.

12. DIARRA I. 2014 : Evaluation de la prise en charge de la malnutrition aigüe sévère des enfants de 06 à 59 mois à I 'URENI du Centre de Santé de Référence de Koutiala. Thèse de Médecine : Bamako ; $2014 ; 87$ p. 13. INSTAT/ MINISTERE DE L'ECONOMIE ET DES FINANCES. 2012 : Enquête nutritionnelle anthropométrique et de mortalité rétrospective : Mali 2012 ; $96 \mathrm{p}$.

14. WIKIPEDIA : Koutiala wikipédia fr.wikipédia.org/wiki/koutiala ; page 123 : 25 Mai 2015 à $20 \mathrm{~h} 62 \mathrm{p}$.

15. MSF : Rapport annuel des activités $2011-2012: 41^{\mathrm{e}}$ assemblée générale, juin 2012.

16. DIARRA B. Evaluation du statut nutritionnel des enfants de 0 à 59 mois dans 4 cercles de la région de Tombouctou. Thèse de Médecine : Bamako ; 2010 ; 90p. 17. KAMATE I. Pratiques alimentaires et suivi nutritionnel des enfants malnutris de 06 à 59 mois dans 2 communes 
du cercle de Sikasso (Kapala et Finkolo). Thèse de pharmacie : Bamako : $2012 ; 62 p$.

18. CLAUDIE S.M. Evaluation du niveau de connaissances, attitudes et pratiques des mères en nutrition et santé de leurs enfants de 06 à 59 mois dans le District sanitaire de Sikasso. Thèse de Médecine : FMPOS Bamako ; 2011 ; 77p.

19. UNICEF. Situation des enfants dans le monde : 2009 ; 125p.

Liste des tableaux :

Tableau I : Analyse des facteurs liés à la malnutrition des 238 enfants

\begin{tabular}{|c|c|c|c|c|}
\hline Facteurs & $\begin{array}{c}\text { Oui } \\
\mathrm{n}(\%)\end{array}$ & $\begin{array}{c}\text { Non } \\
\mathrm{n}\end{array}$ & OR[IC95\%] & $p$ \\
\hline & arasme & & & \\
\hline \multicolumn{5}{|l|}{ Tranche d'âge } \\
\hline 06-24 mois & $81(79,4)$ & 21 & $6,63[3,67-12,01]$ & 0,001 \\
\hline 25 mois et plus & $50(36,8)$ & 86 & 1 & \\
\hline \multicolumn{5}{|l|}{ Sexe } \\
\hline Féminin & $63(51,6)$ & 59 & 1 & \\
\hline Masculin & $68(58,6)$ & 48 & $1,33[0,79-2,21]$ & 0,2803 \\
\hline \multicolumn{5}{|l|}{ Paludisme } \\
\hline Oui & $53(63,1)$ & 31 & $1,67[0,97-2,87]$ & 0,065 \\
\hline Non & $78(50,6)$ & 76 & 1 & \\
\hline \multicolumn{5}{|l|}{ Gastroentérite } \\
\hline Oui & $46(73,0)$ & 17 & $2,87[1,53-5,38]$ & 0,0009 \\
\hline \multirow[t]{2}{*}{ Non } & $85(48,6)$ & 90 & 1 & \\
\hline & washiorkor & & & \\
\hline \multicolumn{5}{|l|}{ Tranche d'âge } \\
\hline 06-24 mois & $11(10,8)$ & 91 & $1,56[0,91-2,68]$ & 0,001 \\
\hline 25 mois et plus & $71(52,2)$ & 65 & 1 & \\
\hline \multicolumn{5}{|l|}{ Sexe } \\
\hline Féminin & $48(39,3)$ & 74 & 1 & \\
\hline Masculin & $34(29,3)$ & 82 & $0,64[0,37-1,1]$ & 0,1042 \\
\hline \multicolumn{5}{|l|}{ Paludisme } \\
\hline Oui & $24(28,6)$ & 60 & 1 & \\
\hline Non & $58(37,7)$ & 96 & $1,51[0,85-2,68]$ & 0,1593 \\
\hline \multicolumn{5}{|l|}{ Gastroentérite } \\
\hline Oui & $13(20,6)$ & 50 & 1 & \\
\hline Non & $69(39,4)$ & 106 & $2,5[1,27-4,95]$ & 0,0073 \\
\hline
\end{tabular}

